\title{
Characterization of the Leaf Color Mutant $h y$ and Identification of the Mutated Gene in Chinese Cabbage
}

\author{
Mengyang Liu, Yin Lu, Shan Wang, Fang Wu, Jingrui Li, Yanhua Wang, Jianjun Zhao', \\ and Shuxing Shen ${ }^{1}$ \\ Key Laboratory of Vegetable Germplasm Innovation and Utilization of Hebei, Collaborative \\ Innovation Center of Vegetable Industry in Hebei, College of Horticulture, Hebei Agricultural \\ University, Baoding, Hebei 071000, People's Republic of China
}

\begin{abstract}
AdDitional INDEX WORDs. chlorophyll-descent, chlorophyll fluorescence, ethyl methanesulfonate mutant, yellow-green leaf
Abstract. Leaf color mutants play an important role in our understanding of chlorophyll biosynthesis and catabolism. In this study, we obtained a yellow-green leaf mutant $h y$ in an ethyl methanesulfonate mutagenized population of chinese cabbage (Brassica rapa ssp. pekinensis). The hy phenotype was controlled by a recessive allele at a single locus. The intrinsic photochemical activity of photosystem II (PSII) is impaired in $h y$, suggesting that absorbed light energy is not efficiently transferred from the light-harvesting complexes antenna to the PSII reaction centers and dissipated as heat or fluorescence. We measured chlorophyll content and chlorophyll precursors and analyzed the expression of key genes in the chlorophyll synthetic pathway in $h y$ and wild type. The mutation phenotype was consistent with inhibited expression of chlorophyll $a$ oxygenase $(C A O)$ gene in the chlorophyll synthetic pathway. In mutant $h y, C A O$ cDNA was cloned so that a $\mathrm{C}$ to $\mathrm{T}$ mutation at $1099 \mathrm{bp}$ caused a conserved proline (Pro) to serine (Ser) mutation at the 367th amino acid in $\mathrm{C}$-domain, which changed the secondary structure of CAO protein. We speculate that the mutation amino acid changed in the $\mathrm{C}$-domain may affect the catalytic function in mutant $C A O$.
\end{abstract}

Leaf color is largely the result of photosynthetic pigments, primarily chlorophyll (Chl). Chl plays an essential role in light absorption for energy transfer (Stern et al., 2004). The Chl biosynthesis pathway is complex and involves more than 20 genes encoding 16 enzymes, and blocking at any step during Chl synthesis can cause changes in leaf color (Beale, 2005).

In higher plants, Chl has two distinct forms, chlorophyll $a(\mathrm{Chl} a)$ and chlorophyll $b(\mathrm{Chl} b)$, that together represent the major light-harvesting pigments in plants. They are associated with Chl $a$, b-binding polypeptides in the peripheral lightharvesting antenna with $\mathrm{Chl} b$ restricted to the light-harvesting complexes (LHC) and is associated with photosystem I (PSI) or PSII (Grossman et al., 1995). Therefore, in the absence of Chl $b$, the LHC proteins are decreased, partly due to the degradation of unbound LHC apoproteins by proteases (Espineda et al., 1999; Hoober and Eggink, 1999). Chl $a$ oxygenase is the critical enzyme for $\mathrm{Chl} b$ synthesis from $\mathrm{Chl} a$ through conversion of a methyl group to a formyl group to generate $\mathrm{Chl} b$ and thus affects the Chl $a / b$ ratio (Espineda et al., 1999; Tanaka et al., 1998). Chl $a$ oxygenase (CAO) converts chlorophyllide a to chlorophyllide $\mathrm{b}$, which is a mononuclear iron-containing

Received for publication 26 Mar. 2018. Accepted for publication 24 July 2018 The authors are grateful to Dr. Kathleen Greenham at Dartmouth College in Hanover, $\mathrm{NH}$, for critical reading of the manuscript.

This work was supported by the National Key R\&D of China (grant 2016YFD0100204-17), the National Natural Science Foundation of China (grant 31672151), the Hundred-Talents Program of Hebei Universities (grant SLRC2017040), the Science and Technology Support Program of Hebei (C2016204170), the Science and Technology Support Program of Hebei (grant 16226304D-2), the Science and Technology Research Project of Hebei Colleges and Universities (grant QN2017084), the International Cooperation project in the Science and Technology Support Program of Hebei (grant17396315D). The Graduate Student Innovation Fund Project in Hebei Province (grant CXZZBS2017067).

${ }^{1}$ Corresponding authors. E-mail: jjz1971@aliyun.com or shensx@hebau.edu.cn. protein via 7-hydroxymethyl chlorophyllide (Oster et al., 2000). CAO is on the chloroplast envelope and acts as the initial site of $\mathrm{Chl} b$ synthesis and LHC assembly (Eggink et al., 2004; Hoober and Eggink, 1999). Overexpression of $C A O$ causes the stimulation of $\mathrm{Chl} b$ synthesis and increased accumulation of the LHCII (Pattanayak et al., 2005; Tanaka et al., 2001). The mRNA and protein expression of $C A O$ is altered by the LHC protein (LHCB), suggesting that enhanced $C A O$ mRNA level affects the size of the LHCII (Biswal et al., 2012; Bujaldon et al., 2017; Harper et al., 2004). In arabidopsis (Arabidopsis thaliana) and rice (Oryza sativa), chlorophyll synthesis is blocked in CAO mutants (Abe et al., 2012; Espineda et al., 1999).

Chinese cabbage is closely related to arabidopsis, providing opportunities for comparative studies of gene function (Trick et al., 2009; Zhao et al., 2005). With the completion of the Brassica rapa genome sequencing and comparative studies with other subspecies, more gene structure information has been acquired (Cheng et al., 2013; Wang et al., 2011). Ethyl methanesulfonate (EMS) is one of the most effective chemical mutagens used to generate single variable point and highdensity mutations that are stably inherited (Henikoff et al., 2004; McCallum et al., 2000). The various mutations are ideal materials for gene function discovery to identify genes involved in key traits in Brassica species (Liu et al., 2010; Stephenson et al., 2010).

In our previous study, an EMS-induced mutant population has been created from $B$. rapa, and several mutants with diverse phenotypes have been identified (Lu et al., 2016). In this study, we focus on one of the EMS-induced mutants, $h y$, that exhibits a yellow-green leaf phenotype with decreased levels of chlorophyll, especially Chl $b$. We identified the leaf color mutant gene through examination of $\mathrm{Chl}$ and its precursors combined with expression data for important genes in the chlorophyll 
synthetic pathway. To examine the impact of the variant $C A O$ alleles, we compared physicochemical and functional properties, secondary structure, and chlorophyll fluorescence in different $C A O$ allele plants. We found changes in the helical and fold structure of CAO, as well as impaired PSII photochemical activity in hy. This screening method for leaf color proved to be an effective method for narrowing down the candidate gene in a population and provides additional material for future studies of the mutated gene function and protein structure.

\section{Materials and Methods}

Plant materials and growth Conditions. The mutant $h y$ was selected from the $\mathrm{M}_{2}$ mutant population, which was obtained from $\mathrm{M}_{1}$ induced from wild-type [WT (A03)] seeds by $0.4 \%$ EMS. hy was selfed strictly to make sure the site was homozygous. The $\mathrm{M}_{4}$ generation mutant was used to cross to WT to obtain $\mathrm{F}_{1}$ progeny, which were self-pollinated to obtain $\mathrm{F}_{2} . \mathrm{F}_{1}$ were backcrossed to the mutant hy to obtain $\mathrm{BC}_{1}$. All the plants were sowed in seeding dishes in Jan. 2016 after 2 months of vernalization at low temperatures $\left(3\right.$ to $\left.8^{\circ} \mathrm{C}\right)$, were grown in a greenhouse and cultivated normally on an experimental farm at Hebei Agricultural University in Baoding, China in Mar. 2016. The phenotypes of hy and WT were observed by visual inspection at the seedling stage $\left(10^{\circ} \mathrm{C}\right)$, the rosette stage $\left(10^{\circ} \mathrm{C}\right)$, and the bolting stage $\left(30^{\circ} \mathrm{C}\right)$. All the samples were collected 1 week after transplanting. We chose the third leaves from inside to outside in the seedling stage for the experiments. Samples for RNA analysis were frozen immediately in liquid nitrogen and stored at $-80{ }^{\circ} \mathrm{C}$.

Measurement of Chl content and precursors. Chls and carotenoids were extracted according to the protocol of Porra et al. (1989) and measured at 663, 646, and $470 \mathrm{~nm}$ with a spectrophotometer (ultraviolet-1800; Shimadzu, Kyoto, Japan). Determination of aminolevulinic acid (ALA), porphobilinogen (PBG), uroporphyrinogen III (Urogen III), and coproporphyrinogen III (Coprogen III) content was based on Bogorad's (1962) methods. ALA, PBG, Urogen III, and Coprogen III were measured at 663, 553, 405.5, and $399.5 \mathrm{~nm}$, respectively.

Precursors, including protoporphyrin IX (Proto IX), magnesium protoporphyrin IX (Mg-Proto IX), and protochlorophyllide (Pchlide), were assayed as described by Rebeiz et al. (1975). Chl precursors were quantified with a fluorescence spectrophotometer (RF-5301PC, Shimadzu) using Ex400:Em632 for Proto IX, Ex440:Em633 for Pchlide and Ex420:Em595 for Mg-Proto IX.

The data were transformed to percentage by setting the WT values to $100 \%$. Three biological and three technical replicates for WT and mutant hy were analyzed. Significance was conducted by $t$ test with percent.

Chlorophyll Fluorescence MEASUREMENT. Photochemical efficiency analysis was performed using a fluorometer (MINI-IMAGINGPAM; Walz, Effeltrich, Germany) from the leaves 1 week after transplanting, according to the manual. The original fluorescence $(F \mathrm{o})$ was induced by measuring light $\left(0.5 \mu \mathrm{mol} \cdot \mathrm{m}^{-2} \cdot \mathrm{s}^{-1}\right.$, frequency $1 \mathrm{~Hz})$ after $1 \mathrm{~h}$ dark adaptation. Pulses $(0.8 \mathrm{~s})$ of red light $\left(5000 \mu \mathrm{mol} \cdot \mathrm{m}^{-2} \cdot \mathrm{s}^{-1}\right)$ were used to determine the maximum fluorescence $(F \mathrm{~m})$. When finished, the pulsed light, actinic light $\left(156 \mu \mathrm{mol} \cdot \mathrm{m}^{-2} \cdot \mathrm{s}^{-1}\right)$, served to induce fluorescence kinetics, steady-state fluorescence $(F \mathrm{~s})$ was measured, and $F \mathrm{~m}^{\prime}$ was determined after exposure to further saturating pulses $(0.8 \mathrm{~s}$, $5000 \mu \mathrm{mol} \cdot \mathrm{m}^{-2} \cdot \mathrm{s}^{-1}$ ) every $20 \mathrm{~s}$.

Photosynthetic parameters were calculated according to previous equations (Genty et al., 1989; Snel and van Kooten, $1990)$, maximum quantum yield of PSII $\left(F_{\mathrm{V}} / F_{\mathrm{m}}\right)=\left(F_{\mathrm{m}}-F_{\mathrm{O}}\right) /$ $F \mathrm{~m}$, photochemical quenching $(\mathrm{qP})=\left(F \mathrm{~m}^{\prime}-F \mathrm{~s}\right) /\left(F \mathrm{~m}^{\prime}-F o\right)$, non-photochemical quenching $(\mathrm{NPQ})=\left(F \mathrm{~m}-F \mathrm{~m}^{\prime}\right) / F \mathrm{~m}^{\prime}$, the effective quantum yields of PSII $(\Phi I I)=\left(F \mathrm{~m}^{\prime}-F\right) / F \mathrm{~m}^{\prime}$, quantum yield of regulatory energy dissipation $[Y(\mathrm{NPQ})]=$ $1-\Phi \mathrm{II}-1 /\left[\mathrm{NPQ}+1+\mathrm{qL}\left(F \mathrm{~m} / F_{\mathrm{O}}-1\right)\right]$, nonquantum yield of regulatory energy dissipation $[Y(\mathrm{NO})]=1 /[\mathrm{NPQ}+1+\mathrm{qL}(F \mathrm{~m} /$ $\left.\left.F_{\mathrm{O}}-1\right)\right]$.

Transcriptional analysis of key genes in Chl BIOSYNTHETIC PATHWAY. Total RNA was isolated using EASY spin Plus (Thermo Fisher Scientific, Waltham, MA), and firststrand cDNA was synthesized via reverse transcription with ReverTra Ace qPCR RT Master Mix (Toyobo, Osaka, Japan). Nucleotide concentration was determined using a spectrophotometer (NanoDrop ND-1000, Thermo Fisher Scientific). Nine related genes associated with $\mathrm{Chl}$ biosynthesis were chosen; primers for the $\mathrm{Chl}$ biosynthesis genes and the reference gene Actin (Act) for the quantitative real-time polymerase chain reaction (RT-qPCR) are listed in Supplemental Table 1. RTqPCR was performed using an RT-PCR detection system (Lightcycler 96; Roche, Basel, Switzerland) following the manufacturer's instructions with specific primer sets using Thunderbird SYBR qPCR Mix (Toyobo). The $2^{-\triangle \triangle \mathrm{CT}}$ method was used to analyze relative change in gene expression (Livak and Schmittgen, 2001). All reactions were performed with three biological and three technical replicates.
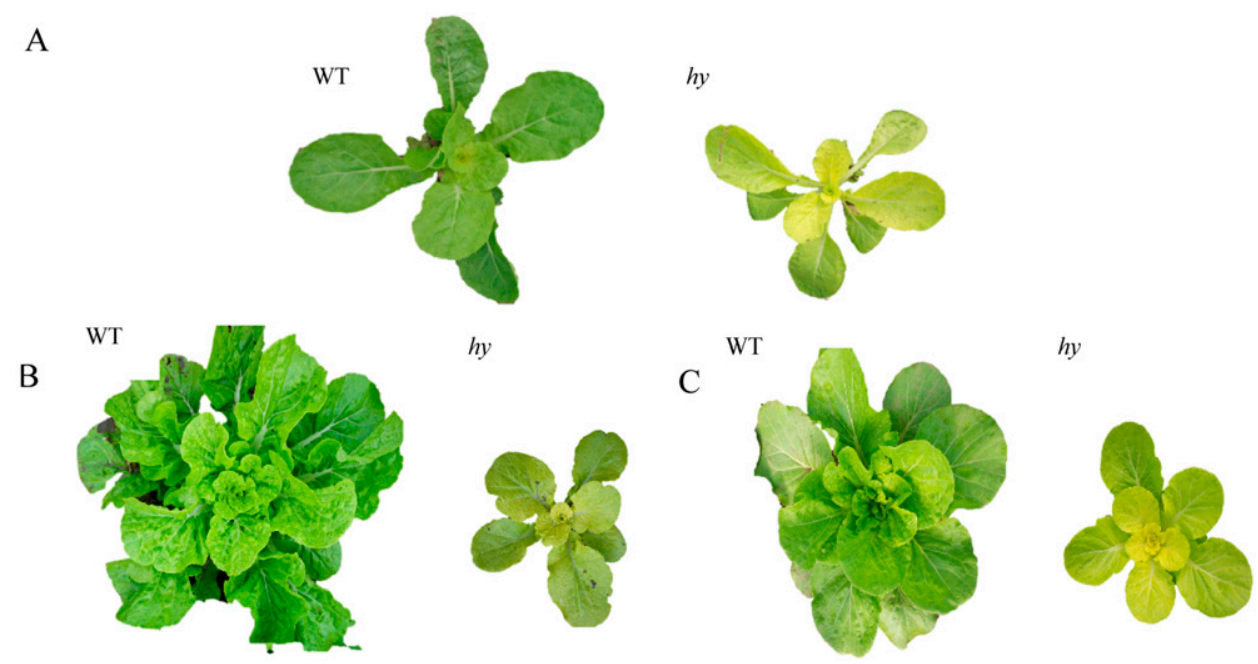

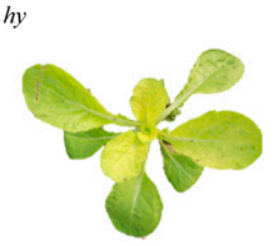

Fig. 1. Phenotype of leaf color of wild type [WT (green leaf)] and hy (yellow-green leaf mutant induced by ethyl methanesulfonate) in chinese cabbage at different temperature and growth stages. (A) Phenotype characteristics of WT and mutant $h y$ at $10^{\circ} \mathrm{C}$ in the seedling stage. (B) Phenotype characteristics of WT and mutant $h y$ at $10^{\circ} \mathrm{C}$ in the rosette stage. (C) Phenotype characteristics of WT and mutant hy at $30^{\circ} \mathrm{C}$ in the bolting stage.

\section{(1)}


CDNA CLONING, SEQUENCE COMPARISON, AND PREDICTION OF PROTEIN STRUCTURE OF THE $\boldsymbol{C A O}$ GENE. Gene annotation within the located region was obtained from the Brassica Database (Wang et al., 2011). cDNA sequences of $C A O$ from the WT and the mutant hy were amplified with the three overlap primers (CAO1, CAO2, and CAO3). The resulting PCR amplicons were extracted and purified using the MiniBEST Agarose Gel DNA Extraction Kit version 4.0 (Takara, Kusatsu, Japan). The products were examined by electrophoresis in a $1 \%$ agarose gel and inserted into the pMD19-T cloning vector (Takara) at $16{ }^{\circ} \mathrm{C}$ overnight followed by transformation into Escherichia coli cells DH5alpha. Positive colonies were selected on LuriaBertani (LB) -amp plates ( $0.1 \%$ ampicillin); single colonies were propagated in LB medium, and the plasmids were extracted and verified by sequencing.

Comparisons of the $C A O$ sequences in $h y$ and WT was performed using DNAMAN 5.0. Homologous sequences of $C A O$ in arabidopsis were identified using the arabidopsis information resource [TAIR (Huala et al., 2001)]. The transit peptide was predicted by the TargetP Server (Emanuelsson et al., 2000). Analysis of the physicochemical properties of amino acid residues and secondary structure in the mutant and WT was performed using

Table 1. Segregation phenotype plant of $F_{2}$ and $\mathrm{BC}_{1}$ population in chinese cabbage with a chi-square test. ${ }^{\mathrm{z}}$

\begin{tabular}{lcc}
\hline Population & $\mathrm{F}_{2}$ & $\mathrm{BC}_{1}$ \\
\hline Green plants (no.) & 164 & 58 \\
Yellow green plants (no.) & 52 & 51 \\
Total plants (no.) & 216 & 109 \\
$\chi^{2}$ & 0.099 & 0.450 \\
$P$ & 0.753 & 0.503
\end{tabular}

${ }^{\mathrm{z}}$ The phenotype of hy mutant is yellow-green leaf induced by ethyl methanesulfonate. The phenotype of wild type (WT) is green leaf. All plants were detected by visual inspection. $P>0.05$ was considered significant. $F_{2}$ plants were self-pollinated $F_{1}$ derived from the cross between the $\mathrm{M}_{4}$ generation mutant and WT. $\mathrm{BC}_{1}$ plants were $\mathrm{F}_{1}$ backcrossed to the mutant hy.

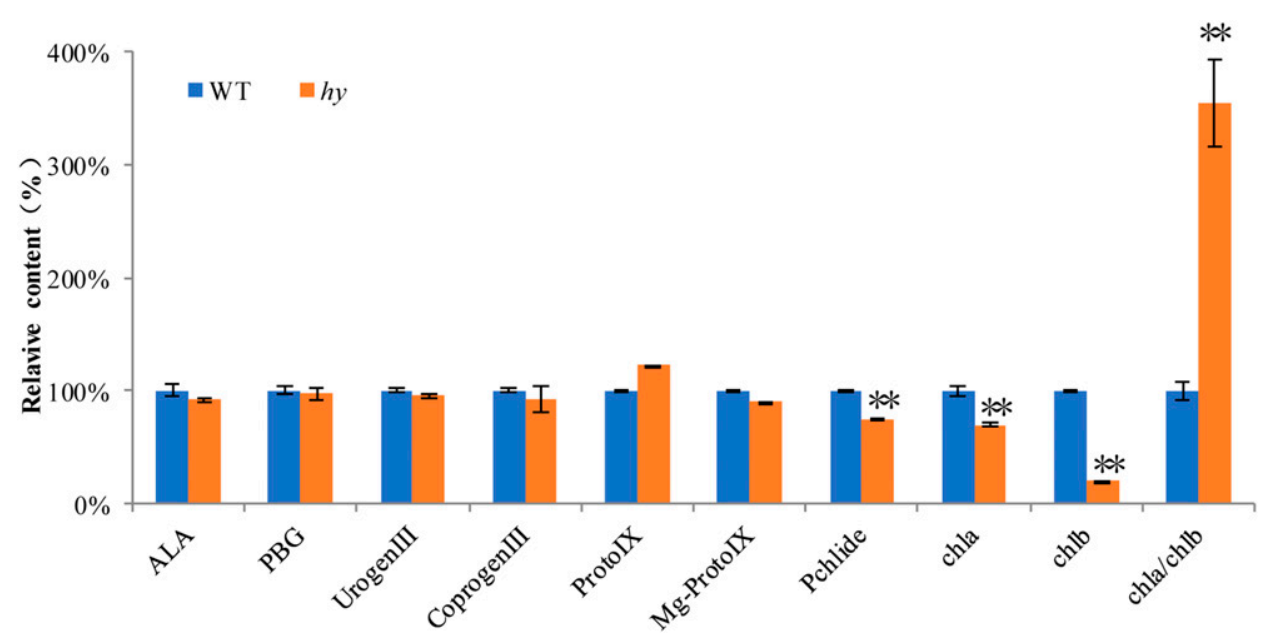

Fig. 2. The contents of chlorophyll biosynthesis precursors and chlorophylls of wild type [WT (green leaf)] and hy (yellow-green leaf mutant induced by ethyl methanesulfonate) in chinese cabbage [** above the vertical bar indicates the differences are significant between the means $(n=3)$ at $P<0.01$ via $t$ test]. ALA $=$ aminolevulinic acid; $\operatorname{chl} a=$ chlorophyll $a$; chl $b=$ chlorophyll $b$; chls $=\operatorname{chl} a$ and $\operatorname{chl} b$; Coprogen III = coproporphyrinogen III; Mg-Proto $=$ Mg-protoporphyrin; $\mathrm{PBG}=$ porphobilinogen; Pchlide $=$ protochlorophyllide; Proto IX = protoporphyrin IX; Urogen III = uroporphyrinogen III.
EXPASY (Gasteiger et al., 1999) and TMHMM (Krogh et al., 2001). Prediction of conserved domains was performed using the NCBI Conserved Domain Search (Marchler-Bauer et al., 2015).

\section{Results}

Phenotypic characterization of the hy mutant. The mutant hy exhibited yellow-green leaves and grew slower than WT that was light green at the seedling stage (Fig. 1A). The leaf color of hy mutants remained a yellow green at $10^{\circ} \mathrm{C}$ during the rosette stage and at the $30^{\circ} \mathrm{C}$ bolting stage (Fig. $1 \mathrm{~B}$ and $\mathrm{C}$ ). The new leaves were yellow green and did not recover with an increase in temperature, which suggests that hy was not sensitive to temperature, and the yellow-green leaves mutant phenotype remains present in different cultural condition at different stages.

Genetic Characterization of THE $\boldsymbol{h y}$ MUtant. $\mathrm{F}_{1}$ plants displayed the WT phenotype, and their $\mathrm{F}_{2}$ population segregation ratio tended to be $3: 1$ [green:yellow-green plants $=164: 52$, $\chi^{2}=0.099, P>0.05$ (Table 1$\left.)\right]$. The $\mathrm{BC}_{1}$ segregation ratio of crosses with hy was 1:1 [green:yellow-green plants $=58: 51$, $\chi^{2}=0.099, P>0.05$ (Table 1)]. This result indicates that the yellow-green phenotype was most likely controlled by a recessive mutation at a single locus.

Quantification OF Chlorophyll and ChL PRECURSORS IN THE hy MUTANT. Chl $a$ level in the mutant hy was $67.4 \%$ lower than WT. Chl $b$ level in the mutant $h y$ was significantly lower at $19.5 \%$ that of WT (Fig. 2). Because of the lower Chl $b$ in the mutant, there was a substantial increase in the Chl $a: b$ ratio compared with WT.

The results showed that the levels of ALA, PBG, Urogen III, Coprogen III, and Mg-Proto IX in the hy mutant were similar to those of WT, the contents were between $90 \%$ and $97 \%$, Proto IX was slightly higher $(122.6 \%)$ than the WT, and Pchlide content was significantly lower $(74.6 \%)$ than in the WT $[P<$ 0.01 (Fig. 2)].

These results suggested that the decrease in Chl was caused by a decrease of Pchlide. Therefore, we speculated that the mutated gene in hy is involved in the conversion of Mg-Proto IX to $\mathrm{Chl} b$.

Chlorophyll Fluorescence of hy Mutant. To further examine the impact of hy mutant on the photosynthetic electron flow and the assembly of PSI and PSII reaction center complexes, we analyzed their chlorophyll fluorescence using PAM techniques (Maxwell and Johnson, 2000). $F \mathrm{~V} / F \mathrm{~m}$ represents the photoenergy conversion efficiency of PSII and initial photoenergy capture efficiency that was 0.731 in hy was similar compared with 0.747 in WT. The Chl fluorescence from PSII showed that $\Phi I I$ in hy was lower than WT. Moreover, the fraction of the photochemical electron transport energy from PSII represented by $\mathrm{qP}$ was decreased in hy compared with WT. The fraction of the nonphotochemical electron transport energy from PSII, NPQ 
was increased in hy compared with WT, which the absorbed light energy that was not dissipated by heat. The photochemical quantum yield by PSII can be divided into two parts, quantum yield of light-induced nonphotochemical quenching $Y(\mathrm{NPQ})$ and quantum yield of nonregulated energy dissipation $Y(\mathrm{NO})$ (Kramer et al., 2004). Although $\Phi I I$ was lower in hy than those in WT, $Y(\mathrm{NPQ})$ was much higher than WT (Table 2 ).

$\Phi$ II and $\mathrm{qP}$ represent the proportion of absorbed energy being used and the proportion of PSII reaction centers that are open, respectively (Genty et al., 1989). These data suggest the photochemical efficiency decrease of PSII resulted in retarded photosynthetic electron transport in hy. $Y(\mathrm{NPQ})$ and $Y(\mathrm{NO})$ were the most important index of PSII energy dissipation and NPQ mechanism also protects against high irradiation (Ögren, 1991). The observed levels suggest that the intrinsic photochemical activity of PSII is impaired in $h y$, and the absorbed light energy is not efficiently transferred from the LHC antenna to PSII reaction centers and dissipated as heat or fluorescence.

EXPRESSION ANALYSIS OF GeNES INVOLVED IN CHL BIOSYNTHESIS IN $\boldsymbol{h y}$ MUTANTS. To identify the mutation accurately, we performed RT-qPCR analysis of some genes associated with $\mathrm{Chl}$ biosynthesis in the WT and hy mutant (Fig. 3). The expression of glutamyl-tRNA reductase (HEMA), porphobilinogen synthase $(H E M B)$, hydroxymethylbilane synthase $(H E M C)$, and uroporphyrinogen decarboxylase (HEME) were slightly downregulated $(80 \%$ to $92 \%)$ in $h y$, suggesting a reduction of glutamyl-tRNA (Glu-tRNA) to Coprogen III conversion in the mutant. The synthesis gene coproporphyrinogen oxidative decarboxylase $(H E M F)$, which is important for conversion of CoprogenIII to Proto IX, was slightly higher in hy $(106.4 \%)$ than in WT. The expression of $\mathrm{Mg}$ chelatase I subunit $(\mathrm{CHLI})$ and $\mathrm{Mg}$-protoporphyrin IX methyltransferase (CHLM), involved in the conversion of Proto IX to Pchlide, were slightly downregulated $(86.6 \%$ and $87.6 \%)$ in $h y$, which also may promote the accumulation of Proto IX. Protochlorophyllide oxidoreductase $(P O R A)$ is the gene in the synthesis of Pchlide to Chl $a$ and was more highly expressed (108.1\%) in hy compared with WT. Nevertheless, these slight changes of expression may not be the reason that the phenotype changed in hy. CAO is the critical enzyme for conversion of Chl $a$ to Chl $b$ and thus affects the Chl $a: b$ ratio (Pattanayak et al., 2005). $C A O$ expression was found to be lower (31\%) in the hy mutant.

The results from the expression analysis of Chl biosynthesisrelated genes support the observed differences in the levels of Chl precursors between hy mutants and WT. On the basis of these results, we considered $C A O$ to be a putative inhibited gene, the expression of which was disrupted in the hy mutant, thereby influencing synthesis of $\mathrm{Chl} b$. However, whether the inhibited expression of $C A O$ was due to function loss or the downstream Chl metabolism-related genes that were acted on remained unknown.

IDENTIFY THE CANDIDATE GENE AND FUNCTIONAL PREDICTION OF THE MUTATED PROTEIN. To understand the $C A O$ change, three pairs of primers (CAO1, CAO2, and CAO3) were used to amplify the $C A O$ full cDNA fragments in mutant hy and WT. The sequencing results showed that there was one mutation in the CAO2 fragment located at the $1099 \mathrm{bp}$ of cDNA causing T to $\mathrm{C}$ change (Supplemental Fig. 1) that corresponds to change from a proline (Pro) to serine (Ser) amino acid in the 367 th codon (Supplemental Fig. 2).

Next, the amino acid sequence of $C A O$ in $h y$ and WT were aligned and compared with those of $A t C A O$ (Fig. 4). These sequences of $C A O$ in hy and WT were highly homologous with the AtCAO, the identity was $96.89 \%$. A sequence of 36 amino acid residues was predicted as a transit peptide using TargetP. The three successive conserved sequences, classified by research of Nagata et al. (2004), were as follows: A-domain, from 37 th to 170 th amino acid, 134 amino acid residues; B-domain, from 171th to 198th amino acid, 28 amino acid residues; C-domain, from 199th to 534th amino acid, 336 amino acid residues. The mutation site was in the C-domain.

We predicted the conserved domains using the National Center for Biotechnology Information Conserved Domain Search and found a conserved Rieske domain from amino acid residues 202 to 330 in the $\mathrm{C}$-domain. This domain contains an iron-sulfur reaction center structure, the alpha subunits contain an electron transfer complex in the N-terminal, whereas the $\mathrm{C}$ terminal plays a catalytic role. There is a predicted START/ RHO_alpha_C/PITP/Bet_v1/CoxG/CalC (SRPBCC) ligandbinding domain at amino acid residues 406 to 499 . The SRPBCC domain has a hydrophobic ligand-binding capsule for binding various ligands. The mutated amino acid is located between these two domains.

The secondary structure of the CAO protein consists of four motifs, including an alpha helix, extended strand, beta turn, and random coil (Supplemental Fig. 3). The number of random coils in the protein structure was predicted to be reduced from $40.45 \%$ to $40.26 \%$ in the hy mutant, and the number of amino acid residues was reduced from 216 to 215 . The alpha helix was predicted to increase from $30.52 \%$ to $30.90 \%$, and the number of amino acid residues was increased from 163 to 165 . The proportion of extended strand was unchanged (19.85\%), and the number of amino acid residues was 106. The proportion of beta turn was the smallest of the four motifs and in the mutant was predicted to be reduced from $9.18 \%$ to $8.99 \%$ with the number of amino acid residues decreasing from 49 to 48 .

Table 2. Chlorophyll fluorescence parameters of wild type [WT (green leaf)] and hy (yellow-green leaf mutant induced by ethyl methanesulfonate) in chinese cabbage.

\begin{tabular}{|c|c|c|c|c|c|c|}
\hline & $F \mathrm{v} / F \mathrm{~m}^{\mathrm{z}}$ & $\Phi I I$ & $Y(\mathrm{NPQ})$ & $Y(\mathrm{NO})$ & NPQ & $\mathrm{qP}$ \\
\hline Phenotype & \multicolumn{6}{|c|}{$($ mean $\pm \mathrm{SE})$} \\
\hline WT & $0.747 \pm 0.001$ & $0.661 \pm 0.005$ & $0.004 \pm 0.002$ & $0.346 \pm 0.016$ & $0.000 \pm 0.000$ & $0.915 \pm 0.015$ \\
\hline hy & $0.731 \pm 0.005^{* *}$ & $0.542 \pm 0.047^{*}$ & $0.093 \pm 0.009 * *$ & $0.365 \pm 0.045$ & $0.289 \pm 0.022 * *$ & $0.855 \pm 0.011^{* *}$ \\
\hline
\end{tabular}

${ }^{\mathrm{z}} \mathrm{F}_{\mathrm{V}} / F \mathrm{~m}=$ maximum quantum yield of PSII; $\Phi \mathrm{II}=$ the effective quantum yields of PSII; $\mathrm{qP}=$ photochemical quenching; NPQ $=$ nonphotochemical quenching; $Y(\mathrm{NPQ})=$ quantum yield of regulatory energy dissipation; $Y(\mathrm{NO})=$ nonquantum yield of regulatory energy dissipation.

Differences significant between the means $(n=3)$ at $* P<0.05$ and at $* * P<0.01$ via $t$ test. 


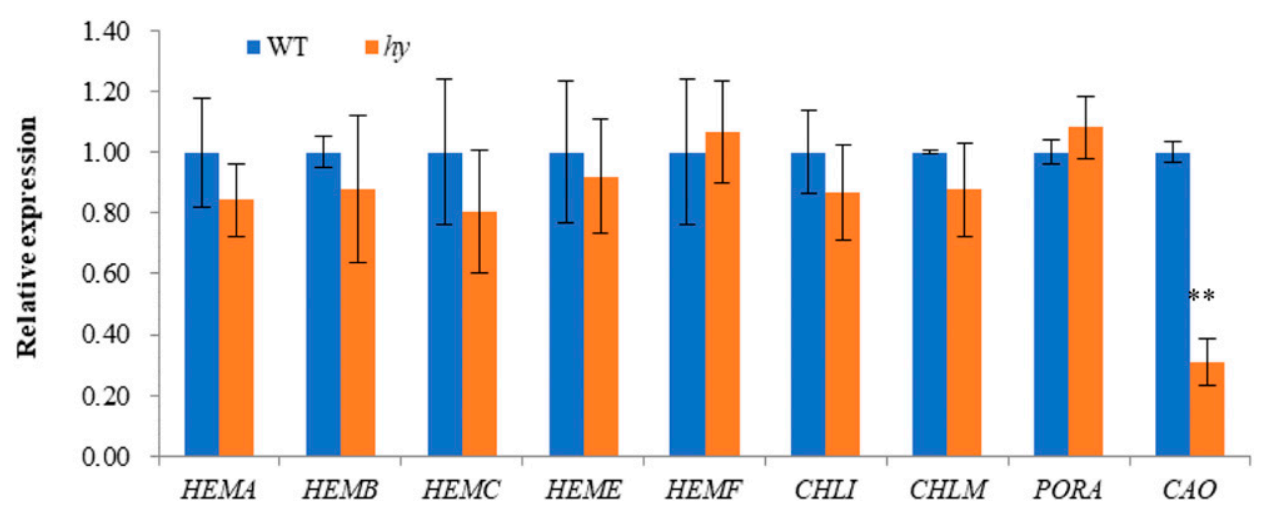

Fig. 3. Expression of chlorophyll synthesis genes of wild type [WT (green leaf)] and hy (yellow-green leaf mutant induced by ethyl methanesulfonate) in chinese cabbage [** above the vertical bar indicates the differences extremely significant between the means $(n=3)$ at $P<0.01$ via $t$ test]. $H E M A=$ glutamyl-tRNA reductase; $H E M B=$ porphobilinogen synthase; $H E M C=$ hydroxymethylbilane synthase; $H E M E=$ uroporphyrinogen decarboxylase; $H E M F=$ coproporphyrinogen oxidative decarboxylase; $C H L I=\mathrm{Mg}$ chelatase I subunit; $C H L M=\mathrm{Mg}$-protoporphyrin IX methyltransferase; $P O R A=$ Protochlorophyllide oxidoreductase; $C A O=$ Chl a oxygenase.

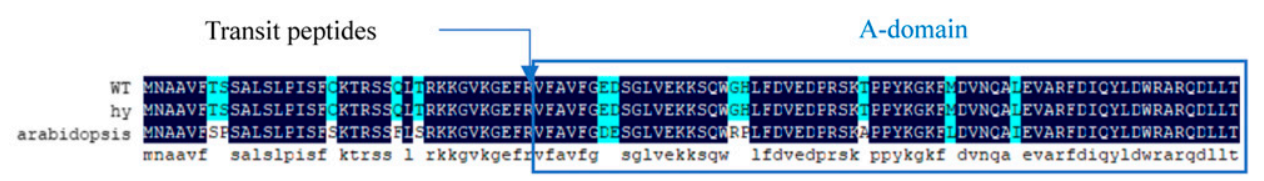

A-domain

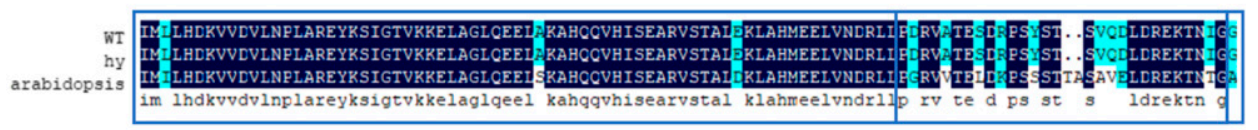

C-domain

Rieske domain
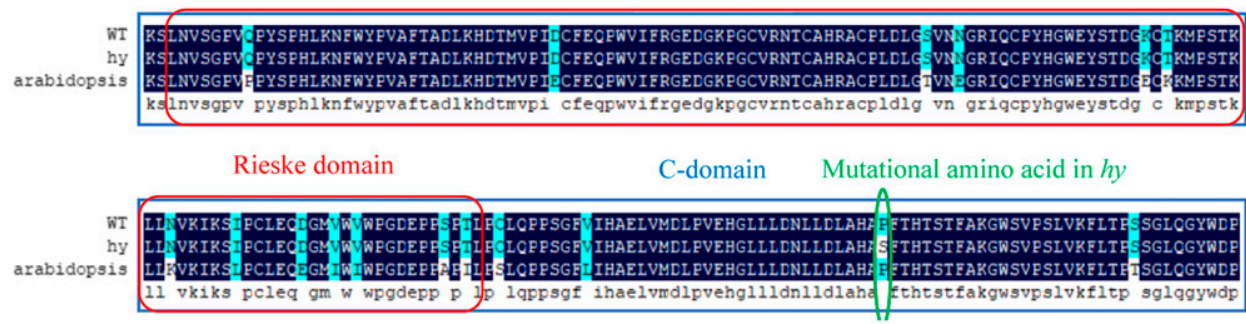

C-domain SRPBCC ligand binding domain

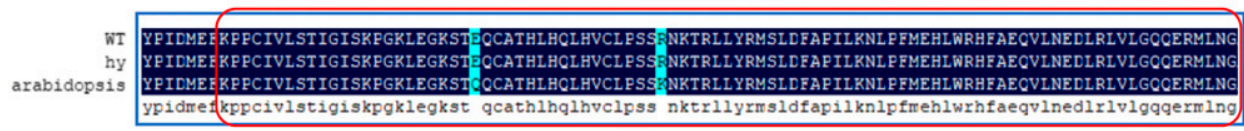

C-domain

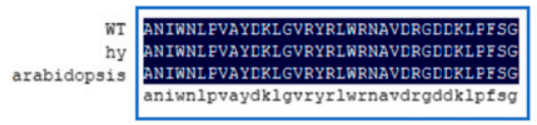

Identity $=96.89 \%$

Fig. 4. Alignment of the amino acid sequence of $\mathrm{Chl}$ a oxygenase $(C A O)$ with three domains of wild type [WT (green leaf)] and hy (yellow-green leaf mutant induced by ethyl methanesulfonate) in chinese cabbage and arabidopsis. The sequences in blue box represent three domains (A-, B-, and C-domain). The sequence before the A-domain is predicted transit peptides. The sequences in the red box represent conserved domains (Rieske domain, SRPBCC ligand-binding domain). The location in the green circle represents the mutational amino acid between WT and hy. The protein sequence of AtCAO and BrCAO has the $96.89 \%$ identity.

\section{Discussion}

THE METHOD OF SCREENING FOR LEAF COLOR MUTANTS. In OUI study, we found that the contents of Chl and the Chl precursors
B-domain

ALA, PBG, Urogen III, Coprogen III, Mg-ProtoIX, and Pchlide were slightly lower in hy than WT, whereas Proto IX was slightly higher in hy than WT. Consistent with these findings, the expression of $H E M F$, the gene responsible for Coprogen III to Proto IX conversion, was increased, and the expression of other genes in the Chl biosynthesis pathway were slightly reduced in $h y$. We speculated that the increased expression of $H E M F$ and the slightly decreased expression of CHLM and CHLI led to increased levels of Proto IX. These results suggest that the levels of $\mathrm{Chl}$ precursors are affected by expression differences in these key genes in the synthesis process. However, the expression of PORA that is required for synthesizing Chl $a$ was only marginally increased in hy $(108.1 \%)$, although the content of Chl $a$ in hy was $67.4 \%$ that of WT. The expression of $C A O$ was significantly lower at $31.1 \%$ of WT, and Chl $b$ was also significantly lower than the WT at $19.5 \%$. Chl $b$ is produced from Chl $a$ by the chlorophyll oxygenase encoded by the CAO gene (Espineda et al., 1999; Tanaka et al., 1998). On the basis of these results, we hypothesized that $C A O$ function was impaired in hy mutant. Sequencing of the gene confirmed a $\mathrm{T}$ to $\mathrm{C}$ mutation corresponding to a change from Pro to Ser amino acid at codon 367 in $h y$. The mutation agrees with typical EMS mutagenesis patterns (McCallum et al., 2000).

At the same time, we speculated that the decrease of Chl $a$ was not caused by Chl $a$ synthesis but by the effects of altered $C A O$ gene function on the chlorophyll cycle. LHC apolipoprotein can combine with chlorophyllide $a$ under the action of CAO to synthesize chlorophyllide $b$ in the thylakoid membrane, and fold after assembly to synthesize Chl $a$ and Chl $b$, but CAO participating in the transportation of LHC apolipoprotein has not been a subject of controversy (Hoober and Eggink, 1999; Tanaka and Tanaka., 2007). In this study, we found that the $C A O$ gene expressed was impaired and the content of Chl $a$ was reduced, suggesting that $C A O$ may influence transport during $\mathrm{Chl} a$ and $\mathrm{Chl} b$ synthesis. Our results also suggested the decrease in photochemical efficiency 
of PSII resulted in retarded photosynthetic electron transport in $h y$. These results suggest that the intrinsic photochemical activity of PSII is impaired in $h y$, and the absorbed light energy is not efficiently transferred from the LHC antenna to PSII reaction centers and dissipated as heat or fluorescence.

$\Phi I I$ and qP are measures of the light energy absorbed by PSII that can be used in photosynthetic electron transport for excitation capability and transfer ability of the reaction center of PSII (Genty et al., 1989). Biswal et al. (2012) suggested that overexpression of $C A O$ in plants leads to an increase in light capture and enhanced ( $40 \%$ to $80 \%)$ electron transport rates of PSI and PSII. Consistent with previous research, our data suggest that the photochemical efficiency decrease of PSII resulted in retarded photosynthetic electron transport in hy. $Y$ (NPQ) and $Y(\mathrm{NO})$ were the most important indices of PSII activity to protect the photosynthetic mechanism. NPQ is a selfprotection mechanism (Ögren, 1991).

These results demonstrate that quantification of chlorophyll and its precursors, combined with the expression of key biosynthetic genes, can effectively reduce the putative genes and be used as a screening method for leaf color mutants.

Suspect PRediction of CAO Mutant PRotein FunCtion in hy. In addition to regulation on the transcriptional level, Chl $b$ synthesis is also regulated by protein stability (Nakagawara et al., 2007). The $C A O$ gene is divided into three domains (ABC) in arabidopsis: the A-domain (134 amino acid residues) and B-domain (30 amino acid) were located at the C-terminal, and the C-domain (337 to 344 amino acid residues) was located at the N-terminal (Nagata et al., 2004). Compared with AtCAO, the sequence of $\mathrm{BrCAO}$ protein had a high identity $[96.89 \%$ (Fig. 4)], so the classification rule of domains was consistent with arabidopsis in hy and WT.

A-domain controls Chl biosynthesis, especially the process of de-etiolation in seedlings (Yamasato et al., 2008). Currently, the function of the B-domain has not been well defined but it has been speculated to be used as the connection of the A- and C-domains (Sakuraba et al., 2007). The Cdomain plays a catalytic role as a unique structure of $\mathrm{CAO}$; when the accumulation of the C-domain by removed A- and B- domains, Chl $a / b$ is decreased from 3.6 to 2.2 (Nagata et al., 2004; Yamasato and Tanaka, 2005). In our study, the Chl $a$ level in the mutant $h y$ was $67.4 \%$ lower than that of WT. Chl $b$ levels in the mutant $h y$ were significantly lower at $19.5 \%$ that of WT. By predicting the conserved domains, we found that there were two conserved domains (Rieske domain, SRPBCC ligand-binding domain) related to catalytic function in the $\mathrm{C}$-domain. The mutation site was in the C-domain, not in one of the conserved sequences, but the mutation amino acid was conserved residue in the $\mathrm{C}$-domain (Nagata et al., 2004). Analysis of the secondary structure models of the mutated protein revealed multiple helical and folding structure effects (Supplemental Fig. 3). To eliminate the transcription effects of the $B r C A O$ promoter, the $1500-\mathrm{bp}$ upstream sequence of $B r C A O$ was cloned; no difference between $h y$ and WT was seen (Supplemental Fig. 4). Therefore, we speculated that the mutation amino acid change affected the catalytic function in the C-domain. The functional deficiency of $C A O$ expression may be due to the change in amino acid in the domain that influences the structure of the C-domain, but further work is needed to verify this prediction.

\section{Conclusions}

Yellow-green leaf EMS-mutated $h y$ was controlled by a recessive allele at a single locus. In mutant $h y$, pchlide, $\mathrm{Chl} a$, and Chl $b$ were significantly decreased, and the intrinsic photochemical activity of PSII was impaired. This resulted in retarded photosynthetic electron transport, suggesting that absorbed light energy is not efficiently transferred from the LHC antenna to the PSII reaction centers and dissipated as heat or fluorescence. Analysis of the expression of key genes in the Chl synthetic pathway indicated that $C A O$ expression was inhibited in $h y$. The $C A O$ gene was cloned, showing that a $\mathrm{C}$ to $\mathrm{T}$ mutation at $1099 \mathrm{bp}$ into cDNA causes a conserved Pro to Ser mutation in the $\mathrm{C}$-domain of CAO protein. We speculate that the mutation amino acid changed in the $\mathrm{C}$-domain may affect catalytic function.

\section{Literature Cited}

Abe, A., S. Kosugi, K. Yoshida, S. Natsume, H. Takagi, H. Kanzaki, H. Matsumura, K. Yoshida, C. Mitsuoka, M. Tamiru, H. Innan, L. Cano, S. Kamoun, and R. Terauchi. 2012. Genome sequencing reveals agronomically important loci in rice using MutMap. Nat. Biotechnol. 30:174-178.

Beale, S.I. 2005. Green genes gleaned. Trends Plant Sci. 10:309312.

Biswal, A.K., G.K. Pattanayak, S.S. Pandey, S. Leelavathi, V.S. Reddy, Govindjee, and B.C. Tripathy. 2012. Light intensity-dependen modulation of chlorophyll $b$ biosynthesis and photosynthesis by overexpression of chlorophyllide $a$ oxygenase in tobacco. Plant Physiol. 159:433-449.

Bogorad, L. 1962. Porphyrin synthesis. Methods Enzymol. 5:885-895. Bujaldon, S., N. Kodama, F. Rappaport, R. Subramanyam, C. de Vitry, Y. Takahashi, and F.A. Wollman. 2017. Functional accumulation of antenna proteins in chlorophyll $b$-less mutants of Chlamydomonas reinhardtii. Mol. Plant 10:115-130.

Cheng, F., T. Mandáková, J. Wu, Q. Xie, M.A. Lysak, and X.W. Wang. 2013. Deciphering the diploid ancestral genome of the mesohexaploid Brassica rapa. Plant Cell 25:1541-1554.

Eggink, L.L., R. LoBrutto, D.C. Brune, J. Brusslan, A. Yamasato, A. Tanaka, and J.K. Hoober. 2004. Synthesis of chlorophyll $b$ : Localization of chlorophyllide $a$ oxygenase and discovery of a stable radical in the catalytic subunit. BMC Plant Biol. 4:5.

Emanuelsson, O., H. Nielsen, S. Brunak, and H.G. Von. 2000. Predicting subcellular localization of proteins based on their Nterminal amino acid sequence. J. Mol. Biol. 300:1005-1016.

Espineda, C.E., A.S. Linford, D. Devine, and J.A. Brusslan. 1999. The $A t C A O$ gene, encoding chlorophyll $a$ oxygenase, is required for chlorophyll $b$ synthesis in Arabidopsis thaliana. Proc. Natl. Acad. Sci. USA. 96:10507-10511.

Gasteiger, E., C. Hoogland, A. Gattiker, S. Duvaud, M.R. Wilkins, R.D. Appel, and A. Bairoch. 1999. Protein identification and analysis tools on the Expasy server. Methods Mol. Biol. 112:571-607.

Genty, B., J.M. Briantais, and N.R. Baker. 1989. The relationship between the quenching of photosynthetic electron transport and quenching of chlorophyll fluorescence. Biochim. Biophys. Acta. 990:87-92.

Grossman, A.R., D. Bhaya, K.E. Apt, and D.M. Kehoe. 1995. Lightharvesting complexes in oxygenic photosynthesis: Diversity, control, and evolution. Annu. Rev. Genet. 29:231-288.

Harper, A.L., S.E. von Gesjen, A.S. Linford, M.P. Peterson, R.S. Faircloth, M.M. Thissen, and J.A. Brusslan. 2004. Chlorophyllide $a$ oxygenase mRNA and protein levels correlate with the chlorophyll $a / b$ ratio in Arabidopsis thaliana. Photosynth. Res. 79:149-159.

Henikoff, S., B.J. Till, and L. Comai. 2004. TILLING, traditional mutagenesis meets functional genomics. Plant Physiol. 135:630636. 
Hoober, J.K. and L.L. Eggink. 1999. Assembly of light-harvesting complex II and biogenesis of thylakoid membranes in chloroplasts. Photosynth. Res. 61:197-215.

Huala, E., A.W. Dickerman, M. Garcia-Hernandez, D. Weems, L. Reiser, F. Lafond, D. Hanley, D. Kiphart, M.Z. Zhuang, W. Huang, L.A. Mueller, D. Bhattacharyya, D. Bhaya, B.W. Sobral, W. Beavis, D.W. Meinke, C.D. Town, C. Somerville, and S.Y. Rhee. 2001. The arabidopsis information resource (TAIR): A comprehensive database and web-based information retrieval, analysis, and visualization system for a model plant. Nucleic Acids Res. 29:102-105.

Kramer, D.M., T.J. Avenson, and G.E. Edwards. 2004. Dynamic flexibility in the light reactions of photosynthesis governed by both electron and proton transfer reactions. Trends Plant Sci. 9:349-357.

Krogh, A., B. Larsson, H.G. Von, and E.L. Sonnhammer. 2001. Predicting transmembrane protein topology with a hidden Markov model: Application to complete genomes. J. Mol. Biol. 305:567-580.

Liu, C., J. Wang, T. Huang, F. Wang, F. Yuan, X. Cheng, Y. Zhang, S. Shi, J. Wu, and K. Liu. 2010. A missense mutation in the VHYNP motif of a DELLA protein causes a semi-dwarf mutant phenotype in Brassica napus. Theor. Appl. Genet. 21:249-258.

Livak, K.J. and T.D. Schmittgen. 2001. Analysis of relative gene expression data using real-time quantitative PCR and the $2^{-} \Delta \Delta \mathrm{CT}$ method. Methods. 25:402-408.

Lu, Y., S.Y. Dai, A.X. Gu, M.Y. Liu, Y.H. Wang, S.X. Luo, Y.J. Zhao, S. Wang, S.X. Xuan, X.P. Chen, X.F. Li, G. Bonnema, J.J. Zhao, and S.X. Shen. 2016. Microspore induced doubled haploids production from ethyl methanesulfonate (EMS) soaked flower buds is an efficient strategy for mutagenesis in chinese cabbage. Front. Plant Sci. 7:1780.

Marchler-Bauer, A., M.K. Derbyshire, N.R. Gonzales, S. Lu, F. Chitsaz, L.Y. Geer, R.C. Geer, J. He, M. Gwadz, D.I. Hurwitz, C.J. Lanczycki, F. Lu, G.H. Marchler, J.S. Song, N. Thanki, Z.X. Wang, R.A. Yamashita, D.C. Zhang, C.J. Zheng, and S.H. Bryant. 2015. CDD: NCBI's conserved domain database. Nucleic Acids Res. 43: D222-D226.

Maxwell, K. and G.N. Johnson. 2000. Chlorophyll fluorescence-A practical guide. J. Expt. Bot. 51:659-668.

McCallum, C.M., L. Comai, E.A. Greene, and S. Henikoff. 2000. Targeted screening for induced mutations. Nat. Biotechnol. 18:455457.

Nagata, N., S. Satoh, R. Tanaka, and A. Tanaka. 2004. Domain structures of chlorophyllide $a$ oxygenase of green plants and Prochlorothrix hollandica in relation to catalytic functions. Planta. 218:1019-1025.

Nakagawara, E., Y. Sakuraba, A. Yamasato, R. Tanaka, and A. Tanaka. 2007. Clp protease controls chlorophyll $b$ synthesis by regulating the level of chlorophyllide $a$ oxygenase. Plant J. 49:800809.

Ögren, E. 1991. Prediction of photoinhibition of photosynthesis from measurements of fluorescence quenching components. Planta. 184:538-544.

Oster, U., R. Tanaka, A. Tanaka, and W. Rudiger. 2000. Cloning and functional expression of the gene encoding the key enzyme for chlorophyll $b$ biosynthesis (CAO) from Arabidopsis thaliana. Plant J. 21:305-310.

Pattanayak, G.K., A.K. Biswal, V.S. Reddy, and B.C. Tripathy. 2005. Light-dependent regulation of chlorophyll $b$ biosynthesis in chlorophyllide $a$ oxygenase overexpressing tobacco plants. Biochem. Biophys. Res. Commun. 326:466-471.

Porra, R.J., W.A. Thompson, and P.E. Kriedemann. 1989. Determination of accurate extinction coefficients and simultaneous equations for assaying chlorophylls $a$ and $b$ extracted with four different solvents: Verification of the concentration of chlorophyll standards by atomic absorption spectroscopy. Biochim. Biophys. Acta. 975:384-394.

Rebeiz, C.A., J.R. Mattheis, B.B. Smith, C.C. Rebeiz, and D.F. Dayton. 1975. Chloroplast biogenesis: Biosynthesis and accumulation of protochlorophyll by isolated etioplasts and developing chloroplasts. Arch. Biochem. Biophys. 171:549-567.

Sakuraba, Y., A. Yamasato, R. Tanaka, and A. Tanaka. 2007. Functional analysis of N-terminal domains of Arabidopsis chlorophyllide $a$ oxygenase. Plant Physiol. Biochem. 45:740-749.

Snel, J.F.H. and O. van Kooten. 1990. The use of chlorophyll fluorescence and other noninvasive spectroscopic techniques in plant stress physiology. Photosynth. Res. 25:147-150.

Stephenson, P., D. Baker, T. Girin, A. Perez, S. Amoah, G.J. King, and L. Østergaard. 2010. A rich TILLING resource for studying gene function in Brassica rapa. BMC Plant Biol. 10:62.

Stern, D.B., M.R. Hanson, and A. Barkan. 2004. Genetics and genomics of chloroplast biogenesis: Maize as a model system. Trends Plant Sci. 9:293-301.

Tanaka, A., H. Ito, R. Tanaka, N.K. Tanaka, K. Yoshida, and K. Okada. 1998. Chlorophyll $a$ oxygenase $(C A O)$ is involved in chlorophyll $b$ formation from chlorophyll $a$. Proc. Natl. Acad. Sci. USA. 95:12719-12723.

Tanaka, R. and A. Tanaka. 2007. Tetrapyrrole biosynthesis in higher plants. Annu. Rev. Plant Biol. 58:321-346.

Tanaka, R., Y. Koshino, S. Sawa, S. Ishiguro, K. Okada, and A. Tanaka. 2001. Overexpression of chlorophyllide $a$ oxygenase (CAO) enlarges the antenna size of photosystem II in Arabidopsis thaliana. Plant J. 26:365-373.

Trick, M., S.J. Kwon, S.R. Choi, F. Fraser, E. Soumpourou, N. Drou, Z. Wang, S.Y. Lee, T.J. Yang, J.H. Mun, A.H. Paterson, C.D. Town, J.C. Pires, Y.P. Lim, B.S. Park, and I. Bancroft. 2009. Complexity of genome evolution by segmental rearrangement in Brassica rapa revealed by sequence-level analysis. BMC Genomics. 10:539.

Wang, X.W., H.Z. Wang, J. Wang, R.F. Sun, J. Wu, S.Y. Liu, Y.Q. Bai, J.H. Mun, I. Bancroft, F. Cheng, S.W. Huang, X.X. Li, W. Hua, J.Y. Wang, X.Y. Wang, M. Freeling, J.C. Pires, A.H. Paterson, B. Chalhoub, B. Wang, A. Hayward, A.G. Sharpe, B.S. Park, B. Weisshaar, B.H. Liu, B. Li, B. Liu, C.B. Tong, C. Song, C. Duran, C.F. Peng, C.Y. Geng, C. Koh, C.Y. Lin, D. Edwards, D.S. Mu, D. Shen, E. Soumpourou, F. Li, F. Fraser, G. Conant, G. Lassalle, G.J. King, G. Bonnema, H.B. Tang, H.P. Wang, H. Belcram, H.L. Zhou, H. Hirakawa, H. Abe, H. Guo, H. Wang, H.Z. Jin, I.A. Parkin, J. Batley, J.S. Kim, J. Just, J.W. Li, J.H. Xu, J. Deng, J.A. Kim, J.P. Li, J.Y. Yu, J.L. Meng, J.P. Wang, J.M. Min, J. Poulain, J. Wang, K. Hatakeyama, K. Wu, L. Wang, L. Fang, M. Trick, M.G. Links, M.X. Zhao, M. Jin, N. Ramchiary, N. Drou, P.J. Berkman, Q.L. Cai, Q.F. Huang, R.Q. Li, S. Tabata, S.F. Cheng, S. Zhang, S.J. Zhang, S.M. Huang, S. Sato, S.L. Sun, S.J. Kwon, S.R. Choi, T.H. Lee, W. Fan, X. Zhao, X. Tan, X. Xu, Y. Wang, Y. Qiu, Y. Yin, Y.R. Li, Y.C. Du, Y.C. Liao, Y. Lim, Y. Narusaka, Y.P. Wang, Z.Y. Wang, Z.Y. Li, Z.W. Wang, Z.Y. Xiong, and Z.H. Zhang. 2011. The genome of the mesopolyploid crop species Brassica rapa. Nat. Genet. 43:10351039.

Yamasato, A. and A. Tanaka. 2005. The N-terminal domain of chlorophyllide $a$ oxygenase confers protein instability in response to chlorophyll $b$ accumulation in Arabidopsis. Plant Cell. 17:1585-1597. Yamasato, A., R. Tanaka, and A. Tanaka. 2008. Loss of the N-terminal domain of chlorophyllide $a$ oxygenase induces photodamage during greening of Arabidopsis seedlings. BMC Plant Biol. 8:64.

Zhao, J.J., X.W. Wang, B. Deng, P. Lou, J. Wu, R.F. Sun, Z.Y. Xu, J. Vromans, M. Koornneef, and G. Bonnema. 2005. Genetic relationships within Brassica rapa as inferred from AFLP fingerprints. Theor. Appl. Genet. 110:1301-1314. 


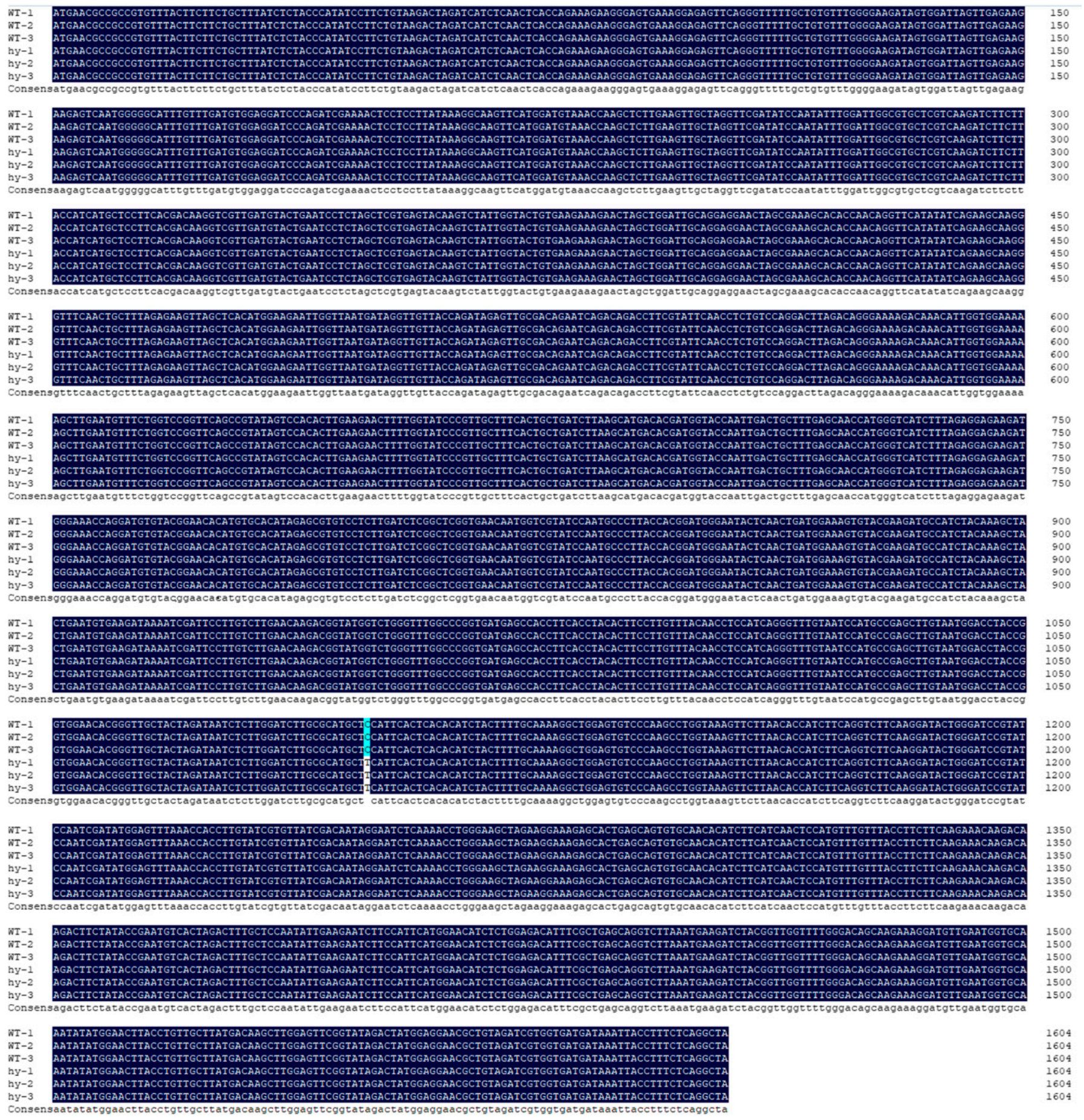

Supplemental Fig. 1. The full cDNA sequence alignment of Chl a oxygenase (CAO) of wild type (WT green leaf) and hy (yellow-green leaf mutant induced by ethyl methanesulfonate) in chinese cabbage. 
Consemnaavftssalslpisfcktrssgltrkkgvkgefrvfavfgedsglvekksgwghlfdvedprsktppykgkfmdvngalevarfdigyldwrargdl

Consetimlihdkvvdvlnplareyksigtvkkelaglgeelakahgqvhisearvstaleklahmeelvndrllpdrvatesdrpsystsvgdldrektniggk

WT SLNVSGPVQPYSPHLKNEWYPVAFTADLRHDTMVPIDCFEQPWVIFRGEDGRPGCVRNTCAHRACPLDLGSVNNGRIQCPYHGWEYSTDGKCTKMPSTRI

hY SLNVSGPVQPYSPHLKNEWYPVAFTADLKHDTMVPIDCFEQPWVIFRGEDGKPGCVRNTCAHRACPLDLGSVNNGRIQCPYHGWEYSTDGKCTKMPSTKL

Conseslnvsgpvgpysphlknfwypvaftadlkhdtmvpidcfegpwvifrgedgkpgcvrntcahracpldlgsvnngrigcpyhgweystdgkctkmpstkl

WT LNVKIRSIPCLEQDGMVWVWPGDEPPSTLPCLQPPSGFVIHAELVMDL PVEHGLLLDNLLDLAHAFFTHTSTFARGWSVPSLVKFLTPSSGLQGYWDPY

hy LNVKIKSIPCLEQDGMVWVWPGDEPPSPTI PCLQPPSGFV IHAELVMDL PVEHGLLLDNLLDLAHZSETHTST FARGWSVPSLVR FLT PSSGLQGYWDPY

Conselnvkiksipclegdgmvwvwpgdeppsptlpclgppsgfvihaelvmdlpvehgllldnlldlaha fthtstfakgwsvpslvkfltpssglggywdpy

\section{WT PIDMEFRPPCIVLST IGISKPGKLEGKSTEQCATHLHQLHVCLPSSRNKTRLIYRMSIDFAPILRNLPFMEHLWRHFAECVINEDLRLVLGQQERMLNGA}

WT NIWNLPVAYDKLGVRYRLWRNAVDRGDDKLPES

hy NIWNLPVAYDKLGVRYRLWRNAVDRGDDKLPFS

Conseniwnlpvaydklgvryrlwrnavdrgddklpfs

Supplemental Fig. 2. The amino acid sequence of Chl a oxygenase (CAO) of wild type (WT green leaf) and hy (yellow-green leaf mutant induced by ethyl methanesulfonate) in chinese cabbage.
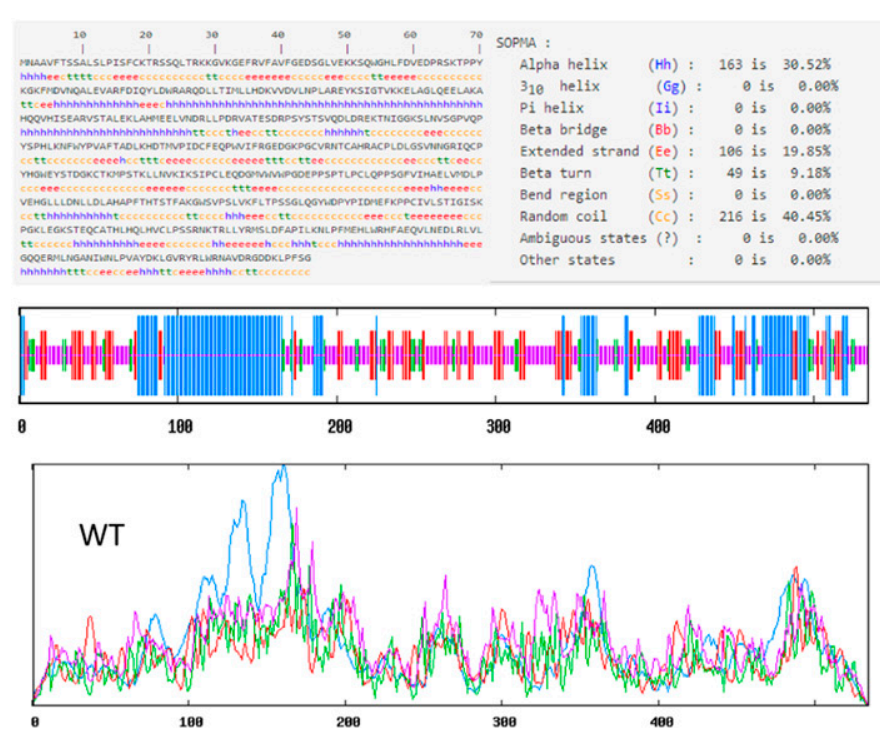

Supplemental Fig. 3. The prediction of Chl a oxygenase (CAO) secondary structure of wild type (WT green leaf) and hy (yellow green leaf mutant induced by ethyl methanesulfonate) in chinese cabbage. The protein of CAO was composed of alpha helix (blue), extended strand (red), beta turn (green), and random coil (yellow). The number and the proportion of components are presented in right of each picture. 


\section{T}

TGCGATTAATCGGGTTTTAGCTGATTAATTGATCTATAATTAATTGAACCGATTTGGCCAAAATTTTGCAATAATCTCCATTGAATGCCCAGTCGGTTAGATACCCACGTTTTAGAGAGAAGTAACAAACTIGTAATATTAAAAAAAA

tgcgattaatcgggtttttagctgattaattgatctataattaattgaaccgatttggccaaaattttgcaataatctccattgaatgcccagtcggttagatacccacgttttagagagaagtaacaaact tgtaatattaaaaaaa

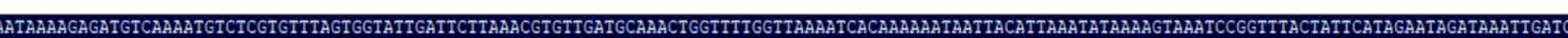

AATAAAAGAGATGTCAAAATGTCTCGTGTTTAGTGGTATTGATTCTTAAACGTGTTGATGCAAACTGGTTTTGGTTAAAATCACAAAAAATAATTACATTAAATATAAAAGTAAATCCGGTTIACTATTCATAGAATAGATAAATTGATC aataaagagatgtcaaatgtctcgtgtttagtggtattgattcttaaacgtgttgatgcaaactggtttggttaaaatcacaaaaataattacattaaatataaaagtaaatccggtttactattcatagaatagataaattgatc

CAAAATAAATTCTTGCACAGTAGAITAITTTTTTAAAAATTCCACCGTAATAAATTTATATTATAATATAGTAGACTATATCAAAAAAACTCTACTGTAATAAATGGTTTAAGATGGCCCTTCATTTTGTGCCTACCTTAGCTGAACTTI

y

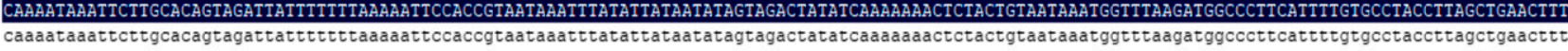

Forward primer of $\mathrm{CAO} 1$

Supplemental Fig. 4. Chl a oxygenase (CAO) putative promoter sequence alignment (1500 bp upstream) of wild type (WT green leaf) and $h y$ (yellow-green leaf mutant induced by ethyl methanesulfonate) in chinese cabbage. The sequence of $C A O$ begins from ATG (the initiation codon) as the blue arrow showed in figure. The sequence of blue parenthesis represents forward primer of CAO1 that was used to amplify the full cDNA of $C A O$. 
Supplemental Table 1. Primers of genes in chlorophyll biosynthesis pathway used for quantitative real-time polymerase chain reaction (RTqPCR) and amplification.

\begin{tabular}{|c|c|c|c|c|c|}
\hline Gene & Gene annotated & Gene ID & Primer sequence $\left(5^{\prime}-3^{\prime}\right)$ & Synthetic pathway & Purpose \\
\hline \multirow[t]{2}{*}{ HEMA } & Glutanyl-tRNA reductase & Bra035447 & F:ATTGGTGTTCGTGCTCTT & Glu-tRNA $\rightarrow$ ALA & RT-qPCR \\
\hline & & & R:ATGTTTGCGGCATTAGGT & & \\
\hline HEMB & Porphobilinogen synthase & Bra036945 & F:AGCCTGAACGCAGCATCGTAT & $\mathrm{ALA} \rightarrow \mathrm{PBG}$ & RT-qPCR \\
\hline \multirow[t]{2}{*}{ HEMC } & Hydroxymethylbilane synthase & Bra005980 & F:TGTCGCATCGGTCTTG & PBG $\rightarrow$ Urogen III & RT-qPCR \\
\hline & & & R:TGTCTCCTCGTGGTTCA & & \\
\hline HEME & Uroporphyrinogen & Bra016989 & F:GACTGGCTAGGACTGGTG & Urogen III $\rightarrow$ Coprogen III & RT-qPCR \\
\hline \multirow[t]{2}{*}{ HEMF } & Coproporphyrinogen oxidative & Bra033402 & F:CGTTCTTTGCTGCGGGAGTTA & Coprogen III $\rightarrow$ Proto IX & RT-qPCR \\
\hline & decarboxylase & & R:GGAACATCCTTTGGAGCATCAGT & & \\
\hline \multirow[t]{2}{*}{ CHLI } & Mg chelatase I subunit & Bra013314 & F:TGTTCAGATTGATAGGGAGT & Proto IX $\rightarrow$ Mg-Proto IX & RT-qPCR \\
\hline & & & R:TCGCTGCTCTGTTAGTCA & & \\
\hline \multirow[t]{2}{*}{ CHLM } & Mg-protoporphyrinIX & Bra019192 & F:AAAGCAGCAGCTAGGAGG & Mg-Proto IX $\rightarrow$ Pchlide & RT-qPCR \\
\hline & methyltransferase & & R:TTTGTTCTGAGGGTAATGT & & \\
\hline \multirow[t]{2}{*}{$\mathrm{CAO} 1$} & Chlorophyllide a oxygenase & Bra036948 & F:ATGAACGCCGCCGTGTTTACTT & & PCR \\
\hline & & & R:TTCCCTGTCTAAGTCCTG & & \\
\hline \multirow[t]{2}{*}{$\mathrm{CAO} 2$} & Chlorophyllide a oxygenase & Bra036948 & F:TTACCAGATAGAGTTGCGACAG & & PCR \\
\hline & & & R:TGGGACACTCCAGCCTTT & & \\
\hline \multirow[t]{2}{*}{$\mathrm{CAO} 3$} & Chlorophyllide a oxygenase & Bra036948 & F:GAACACGGGTTGCTACTA & & PCR \\
\hline & & & R:TTAGCCTGAGAAAGGTAAT & & \\
\hline \multirow[t]{2}{*}{ ACTIN } & Reference gene & $\mathrm{Bra} 028615$ & F:GCTTCCCTCAGCACTT & & RT-qPCR \\
\hline & & & R:GACCCGACTCATCGTAC & & \\
\hline \multirow[t]{2}{*}{ PCAO } & Upstream of CAO & & F:ATCATAAGGAAATGGTCT & & PCR \\
\hline & & & R:GCTGTCGTCACCGGAAGGA & & \\
\hline
\end{tabular}

$\overline{\mathrm{ALA}}=$ aminolevulinic acid; Chl $a=$ chlorophyll $a$; Chl $b=$ chlorophyll $b$; Coprogen III = coproporphyrinogen III; F $=$ forward; Glu-tRNA $=$ glutamyl-tRNA; Mg-Proto = Mg-protoporphyrin; $\mathrm{PBG}=$ porphobilinogen; Pchlide = protochlorophyllide; Proto IX = protoporphyrin IX; $\mathrm{R}=$ reverse; Urogen III = uroporphyrinogen III. 